\title{
The Strong Dodecahedral Conjecture and Fejes Tóth's Conjecture on Sphere Packings with Kissing Number Twelve
}

\author{
Thomas C. Hales ${ }^{\star}$ \\ University of Pittsburgh \\ hales@pitt.edu
}

\begin{abstract}
This article sketches the proofs of two theorems about sphere packings in Euclidean 3-space. The first is K. Bezdek's strong dodecahedral conjecture: the surface area of every bounded Voronoi cell in a packing of balls of radius 1 is at least that of a regular dodecahedron of inradius 1 . The second theorem is L. Fejes Tóth's conjecture on sphere packings with kissing number twelve, which asserts that in 3-space, any packing of congruent balls such that each ball is touched by twelve others consists of hexagonal layers. Both proofs are computer assisted. Complete proofs of these theorems appear in Hal12al and Hal12b.
\end{abstract}

\section{The strong hexagonal conjecture}

To describe methods, we begin with a proof of the following elementary computerassisted theorem in $\mathbb{R}^{2}$.

Theorem 1. The perimeter of any bounded Voronoi cell of a packing of congruent balls of radius 1 in $\mathbb{R}^{2}$ is at least $4 \sqrt{3}$, the perimeter of a regular hexagon with inradius 1 .

If we adopt the convention that the perimeter of an unbounded Voronoi cell is infinite, then the boundedness hypothesis can be dropped from the theorem.

Proof. Fix a bounded Voronoi cell in a packing of congruent balls of radius 1 and fix a coordinate system with the center of the Voronoi cell at the origin. The intersection of the Voronoi cell with a disk of radius $\sqrt{2}$ at the origin is a convex disk whose boundary $C$ consists of circular arcs and straight line segments. The length of $C$ is no greater than the original perimeter of the Voronoi cell. It suffices to show that the length of $C$ is at least $4 \sqrt{3}$.

The boundary $C$ is determined by the set of centers $V$ of balls at distance less than $\sqrt{8}$ from the origin, excluding the ball centered at the origin.

The following piecewise linear function arises in the proof of the strong dodecahedral conjecture in three-dimensions. We make repeated use of it.

$$
L(h)= \begin{cases}\left(h_{0}-h\right) /\left(h_{0}-1\right), & \text { if } h \leq h_{0}, \\ 0, & \text { otherwise }\end{cases}
$$

\footnotetext{
* Research supported by NSF grant 0804189 and the Benter Foundation.
} 
where $h_{0}=1.26$.

Let $\mathbf{u}_{1}, \mathbf{u}_{2} \in V$ be distinct points such that $T=\left\{\mathbf{0}, \mathbf{u}_{1}, \mathbf{u}_{2}\right\}$ has circumradius less than $\sqrt{2}$. Let $\ell\left(\mathbf{u}_{1}, \mathbf{u}_{2}\right)$ be the length of the part of $C$ contained in the convex hull of $T$, and let $\theta$ be the angle at $\mathbf{0}$ between $\mathbf{u}_{1}$ and $\mathbf{u}_{2}$ (see Figure 1 1 a). The following inequality has been verified by computer using interval arithmetic.

$$
\left.\ell\left(\mathbf{u}_{1}, \mathbf{u}_{2}\right)-b \theta\left(\mathbf{u}_{1}, \mathbf{u}_{2}\right)-c L\left(\left\|\mathbf{u}_{1}\right\| / 2\right)-c L\left(\left\|\mathbf{u}_{2}\right\| / 2\right)\right) \geq 0,
$$

where $b=4 / 3$ and $c=\sqrt{3} / 3-2 \pi / 9 \approx-0.12$. Equality holds when $T$ is an equilateral triangle with side 2 .

Remark 1. Let $\mathbf{u}_{1}(t)$ and $\mathbf{u}_{2}(t)$ be points such that

$$
\left\|\mathbf{u}_{1}(t)\right\|=\left\|\mathbf{u}_{2}(t)\right\|=2, \quad\left\|\mathbf{u}_{1}(t)-\mathbf{u}_{2}(t)\right\|=t,
$$

that is a triangle at the origin with sides 2,2,t. Along this curve with parameter $t$, the constants $b$ and $c$ are the unique choice of constants that give the left-hand side of (2) a local minimum with value 0 at $t=2$.
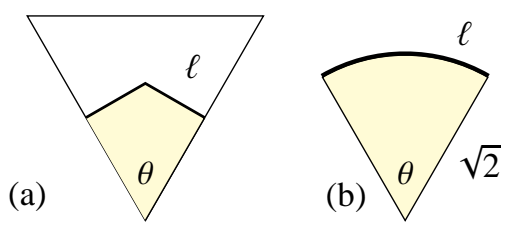

(c)

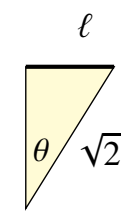

Fig. 1. The truncated boundary of the Voronoi cell (shaded) is partitioned into three types of pieces, indicated with a thick curve marked $\ell$.

The entire boundary $C$ can be partitioned into finitely many (a) pieces lying in convex hulls of triangles $T$, (b) arcs of circles of radius $\sqrt{2}$, and (c) linear segments from $\mathbf{u} / 2$, where $\mathbf{u} \in V$, to a point on the circle of radius $\sqrt{2}$ centered at the origin (Figure1).

We extend the inequality (2) to boundary arcs of type (b) in the form

$$
\ell-b \theta \geq 0
$$

where $\ell$ is the length of the circular arc and $\theta$ is the subtended angle. This inequality is obvious, because $\ell=\sqrt{2} \theta$, and $\sqrt{2}>b=4 / 3$. We extend the inequality (2) to segments of type (c) in the form

$$
\ell(\mathbf{v})-b \theta(\mathbf{v})-c L(h) \geq 0,
$$

where $h=\|\mathbf{v}\| / 2, \theta$ is the subtended angle at the origin, and $\ell=\sqrt{2-h^{2}}$ is the length of the the segment. When $h \leq h_{0}$, inequality (4) is a consequence of inequality (2), because the segment can be reflected through a mirror to form the two segments in (2). When $h \geq h_{0}$, the term $L(h)$ is zero. In this case, basic calculus gives the inequality. 
Let $\ell_{C}$ be the length of $C$. We sum the inequalities over the boundary pieces of $C$ of types (a), (b), and (c), using inequalities (2), (3), and (4):

$$
\ell_{C}-b(2 \pi)-2 c \sum_{\mathbf{v} \in V} L(\|\mathbf{v}\| / 2) \geq 0 .
$$

The function $L$ is zero on $\left\{h: h \geq h_{0}\right\}$. We drop such terms from the sum. Lemma1 and Inequality (5) give

$$
\ell_{C} \geq 2 \pi b+12 c=4 \sqrt{3} \text {. }
$$

This proves the theorem.

The following lemma is used in the proof

Lemma 1. Let $V$ be a set of points contained in a closed annulus at the origin of inner radius 2 and outer radius $2 h_{0}$. Assume that the mutual separation of points in $V$ is at least 2. Then

$$
\sum_{\mathbf{v} \in V} L(\|\mathbf{v}\| / 2) \leq 6 .
$$

Equality is obtained uniquely when $V$ is the set of extreme points of a regular hexagon of circumradius 2 .

Proof. In case $\operatorname{card}(V) \leq 6$, by the inequality $L(\|\mathbf{v}\| / 2) \leq 1$, it is clear that the sum is at most 6 , with equality uniquely obtained for the regular hexagon of circumradius 2 . An easy estimate shows that the angles at the origin between $\mathbf{u}, \mathbf{v} \in V$ is greater than $\pi / 4$, so that $\operatorname{card}(V) \leq 7$. We may therefore assume without loss of generality that $\operatorname{card}(V)=7$.

We index the 7 points $\mathbf{v}_{i}$ by $i \in \mathbb{Z} / 7 \mathbb{Z}$ in their natural cyclic order around the annulus. Let $\theta_{i}$ be the angle subtended at the origin between $\mathbf{v}_{i}$ and $\mathbf{v}_{i+1}$. Let

$$
\alpha_{i}=\arccos (\|\mathbf{v}\| / 4)-\pi / 6 .
$$

We have the following inequality

$$
\theta_{i} \geq \alpha_{i}+\alpha_{i+1}, \quad i \in \mathbb{Z} / 7 \mathbb{Z},
$$

which is proved by basic calculus: it follows from the intermediate value theorem and from an explicit analytic formula for the terms in the inequality [Hal12a, Lemma 6.107]. Further, we have

$$
\alpha_{i}-0.16 L\left(\left\|\mathbf{v}_{i}\right\| / 2\right)-0.32 \geq 0
$$

which is also proved by basic calculus: by a second derivative test the left-hand side is concave as a function of $\left\|\mathbf{v}_{i}\right\|$ so that the inequality holds if it holds at the endpoints $\left\|\mathbf{v}_{i}\right\|=2,2.52$, which is easily checked.

Summing $\theta_{i}$ over $i$ we get

$$
2 \pi=\sum_{i} \theta_{i} \geq 2 \sum_{i} \alpha_{i} \geq 2(0.16) \sum_{i} L\left(\left\|\mathbf{v}_{i}\right\| / 2\right)+14(0.32) .
$$

Computing constants, we get

$$
6>\sum_{i} L\left(\left\|\mathbf{v}_{i}\right\| / 2\right) .
$$


Remark 2. The proof can be organized in a way that carries over directly from two dimensions to three. In the first step (Lemma1), $\sum L(\cdot)$ is shown to be at most the kissing number (which is 6 in dimension two and 12 in dimension three). In the second step, the estimate of the boundary of the truncated Voronoi cell is transformed into an estimate of $\sum L(\cdot)$. The second step can be broken into two smaller steps: (a) use a simplex whose circumradius is less than $\sqrt{2}$ to design an inequality with a local minimum at the desired solution of to the Voronoi cell problem; (b) extend the inequality from part (a) so that it holds on a full geometric partition of the boundary of the truncated Voronoi cell. In a final short step, sum all the inequalities to obtain the desired result.

\section{Marchal cells}

In this section, we give details of the partition of the boundary $C$ of the truncated Voronoi cell. The partition is based on the partition of Euclidean space into Marchal cells [Mar09].

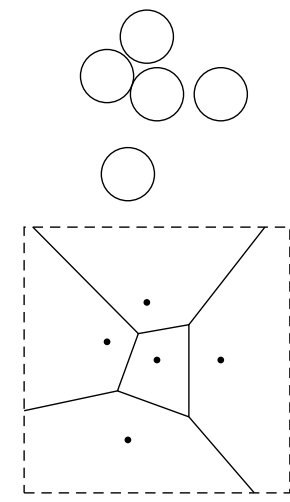

Voronoi cells

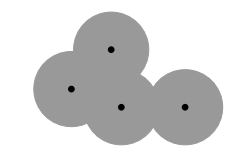

$\mathrm{level}_{\geq 1}$

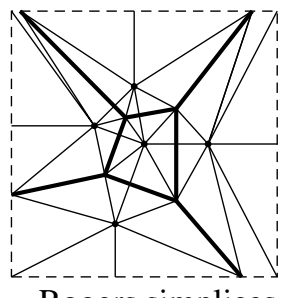

Rogers simplices

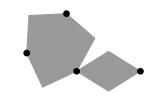

- $\quad \mathrm{level}_{\geq 2}$

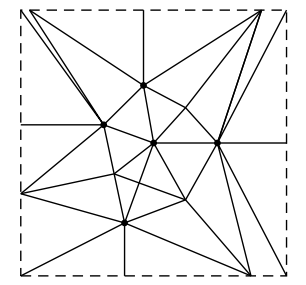

$k$ ! composites

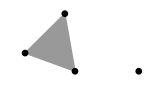

- level $_{\geq 3}$

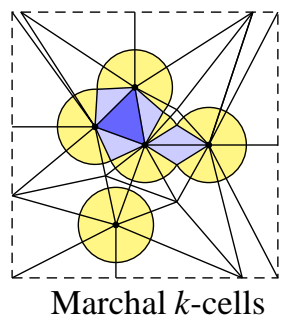

Fig. 2. Partitions of the plane (image source [Hal12a]).

Figure 2 shows a packing $V$ of cardinality five. We use the constant $\sqrt{2}$ to partition the plane into levels $0, \ldots, 3$. Every point has level $\geq 0$. For every $\mathbf{v} \in V$, we form a closed disk of radius $\sqrt{2}$. A point at level $\geq 1$ is a point that lies inside some such disk. We form a closed rhombus of side $\sqrt{2}$ for every pair of distinct points in $V$ whose separation is less than $\sqrt{8}$. By construction, the two points in $V$ are opposite vertices of the rhombus. A point of level $\geq 2$ is a point that lies some rhombus. We form a closed triangle for every triple of distinct points in $V$ whose circumradius is less than $\sqrt{2}$. A point of level 3 is a point that lies inside some such triangle. No point has level $\geq 4$. A point of level $k$ is a point of level $\geq k$ that does not have level $\geq k+1$.

The points of a given level can be further partitioned using the Rogers partition of the plane into simplices [Rog58]. For each $k=0, \ldots, 3$, Rogers simplices that meet the 
set of level $k$ can be naturally grouped into collections of $k$ ! simplices. If $P$ is the union of the $k$ ! simplices, then the set of points of level $k$ in $P$ is called a Marchal $k$-cell $P_{k}$.

The construction can be generalized to three or more dimensions, again using the parameter $\sqrt{2}$. In $n$ dimensions, the levels extend from 0 to $n+1$ in an analogous manner. Let $S \subset V$ be a set of cardinality $k+1$ whose circumradius is less than $\sqrt{2}$. The shapes used to define level sets are the convex hulls of

$$
S \cup X_{S}
$$

where $X_{S}$ is the set of points at equidistance $\sqrt{2}$ from every point of $S$. The set $X_{S}$ is sphere of dimension $n-\operatorname{card}(S)$. When $n=3$, the shapes are balls of radius $\sqrt{2}$, bicones, bipyramids, and tetrahedra (Figure 3). Again in higher dimensions, the Rogers simplices can be grouped into collections of $k$ ! simplices, giving Marchal $k$-cells $P_{k}$, at each level $k$.

\begin{tabular}{lll}
\hline $\operatorname{card}(S)$ & $X_{S}$ & $\operatorname{conv}\left(S \cup X_{S}\right)$ \\
\hline & & \\
1 & sphere & ball \\
2 & circle & bi-cone \\
3 & pair of points & bi-pyramid \\
4 & $\varnothing$ & simplex \\
\hline
\end{tabular}

Fig. 3. Convex hulls used to construct level sets in three dimensions

Remark 3. Marchal introduced cells to show that the Kepler conjecture in three-dimensions can be reduced to an inequality of the form

$$
\sum_{\mathbf{v} \in V} M(\|\mathbf{v}\|) \leq 12
$$

where $M$ is a certain quartic polynomial, and $V$ is a finite packing contained in a closed annulus of inner radius 2 and outer radius $\sqrt{8}$.

Remark 4. The book [Hal12a] strengthens Marchal's argument to reduce the Kepler conjecture to the inequality

$$
\sum_{\mathbf{v} \in V} L(\|\mathbf{v}\| / 2) \leq 12,
$$

where $L$ is the function defined above, and $V$ is a packing in the closed annulus with inner radius 2 and outer radius $2 h_{0}$. (In adapting this inequality from dimension two to dimension three, the two-dimensional kissing number 6 is replaced with the threedimensional kissing number 12.) The book also gives a computer-assisted proof of the inequality $\left[L_{12}\right)$, to obtain a new proof of the Kepler conjecture. This article shows how to deduce the strong dodecahedral conjecture and Fejes Tóth's conjecture on packings with kissing number twelve from $\left(L_{12}\right)$. 
Remark 5. An old conjecture by L. Fejes Tóth [Fej72, p. 178] asserts that the minimum of

$$
\sum_{\mathbf{v} \in V}\|\mathbf{v}\|
$$

is $24+14 / \sqrt{27} \approx 26.69$ as $V$ runs over packings of cardinality 13 contained in a closed annulus with inner radius 2 and outer radius $\sqrt{8}$. The inequality $\left(L_{12}\right)$ gives the best known result:

$$
\sum_{\mathbf{v} \in V}\|\mathbf{v}\| \geq 24+2 h_{0}=26.52 .
$$

The inequality $\left(L_{12}\right)$ also gives upper bounds for the Tammes problem when $\operatorname{card}(V)=$ $13,14,15$, but these upper bounds are weaker than those known by semi-definite programming [BV08].

\section{Strong Dodecahedral Conjecture}

This section sketches a proof of the strong dodecahedral conjecture [Bez00]:

Theorem 2. The surface area of every bounded Voronoi cell in a packing of balls of radius 1 is at least the surface area of a regular dodecahedron of inradius 1. Equality is obtained exactly when the bounded Voronoi cell is itself a regular dodecahedron.

Remark 6. Fejes Tóth's classical dodecahedral conjecture [Fej43] is the same statement, replacing surface area with volume. The classical dodecahedral conjecture was proved by McLaughlin [HM10]. To deduce the volume statement from the surface area statement, it is enough to use the volume formula $B h / 3$ for a tetrahedron, where $B$ is its base area (the face of a Voronoi cell), and $h \geq 1$ is its height.

Proof. We pick coordinates so that the center of a chosen bounded Voronoi cell is at the origin. As in the two-dimensional case, we may intersect the Voronoi cell with a closed ball of radius $\sqrt{2}$. The boundary $C$ after truncation is no greater than before. Let $V$ be the set of centers of the packing in the annulus with inner radius 2 and outer radius $\sqrt{8}$.

There is a partition $C \cap P_{k}$ of $C$ associated with the set of Marchal $k$-cells $P_{k}$ associated with Rogers simplices at the origin. Write

$$
\operatorname{area}(C)=\sum_{P_{k}} \operatorname{area}\left(C \cap P_{k}\right),
$$

for the areas of the various contributions. Write $\operatorname{sol}\left(P_{k}\right)$ for the solid angle of the Marchal cell at the origin, and write $\operatorname{dih}\left(P_{k}, \mathbf{v}\right)$ for the dihedral angle of a Marchal cell $P_{k}$ along the edge through the line through $\{\mathbf{0}, \mathbf{v}\}$.

As a reference cell, we let $P_{D, 4}$ be a Marchal 4-cell of the packing giving the regular dodecahedron of inradius 1 . There exist constants $a_{D}$ and $b_{D}>0$ such that

$$
\operatorname{area}\left(C \cap P_{k}\right)+3 a_{D} \operatorname{sol}\left(P_{k}\right)+3 b_{D} \sum_{\mathbf{v} \in P_{k} \cap V} L(\|\mathbf{v}\| / 2) \operatorname{dih}\left(P_{k}, \mathbf{v}\right) \geq 0
$$


for all $k$-cells $P_{k}$ and for all $V$. The constants $a_{D} \approx-0.581$ and $b_{D} \approx 0.0232$ are uniquely determined if we insist that equality is attained when $P_{k}=P_{D, 4}$. This inequality has been proved by computer by interval arithmetic.

In more detail, the constants $a_{D}$ and $b_{D}$ are determined by a 1-dimensional family $P_{4}(t)$ of tetrahedra with sides $2,2,2, t, t, t$, for $t \in \mathbb{R}$ where the three edges of length 2 meet at the origin. When $t=t_{D} \approx 2.1029$ (the separation of balls in the arrangement giving the regular dodecahedron), $P_{4}\left(t_{D}\right)$ is congruent to $P_{D, 4}$. Forcing the equality to be exact and the derivative to vanish when $t=t_{D}$, we obtain two linear equations in two unknowns that determine $a_{D}$ and $b_{D}$.

If we sum (10) over all cells, the solid angles sum to $4 \pi$, dihedral angles sum to $2 \pi$, and $L$ sums to at most 12 by Ineq. ( $L_{12}$, giving

$$
\operatorname{area}(C)=\sum_{P_{k}} \operatorname{area}\left(C \cap P_{k}\right) \geq-3 a_{D} 4 \pi-3 b_{D}(2 \pi)(12) \text {. }
$$

By the choice of $a_{D}$ and $b_{D}$, equality is obtained for the boundary $C_{D}$ of the regular dodecahedron,

$$
\operatorname{area}\left(C_{D}\right)=-3 a_{D} 4 \pi-3 b_{D}(2 \pi)(12) .
$$

Hence area $(C) \geq \operatorname{area}\left(C_{D}\right)$. This is the desired conclusion. (The circumradius of the regular dodecahedron is less than $\sqrt{2}$ so that $C_{D}$ is both the truncated and untruncated boundary.) Tracing through the case of equality, inequality (10) is an equality exactly when the cell has measure zero or is congruent to $P_{D, 4}$.

\section{Fejes Tóth's Conjecture on Packings with Kissing Number Twelve}

L. Fejes Tóth conjectured the following result in 1969 [Fej69], [Fej89].

Theorem 3. In 3-space any packing of equal ball such that each ball is touched by twelve others consists of hexagonal layers.

The proof of this theorem is much longer than the proof of the strong dodecahedral conjecture. This section describes the proof strategy. The details are found in [Hal12b].

It is enough to prove that the contact pattern of every ball is the hexagonal-close packing (HCP) or face-centered cubic (FCC) kissing arrangement, because these can only be extended in hexagonal layers. In fact, the HCP piece has a preferred plane of symmetry. Once a single HCP piece occurs, the preferred plane must be filled with HCP pieces. A plane forces another hexagonal layer above it and another hexagonal layer below it, leading to a packing of hexagonal layers. If no HCP piece occurs, the packing is the face-centered cubic packing, which also consists of hexagonal layers.

Lemma 2. Let $V$ be a packing in which every ball touches twelve others. Then for all distinct $\mathbf{u}, \mathbf{v} \in V$, either $\|\mathbf{u}-\mathbf{v}\|=2$ or $\|\mathbf{u}-\mathbf{v}\| \geq 2 h_{0}$. 
Proof. Let $\mathbf{u}_{1}, \ldots, \mathbf{u}_{12}$ be the twelve kissing points around $\mathbf{u}$. Assume that $\mathbf{v} \neq \mathbf{u}_{i}, \mathbf{u}$. By Inequality $\left[L_{12}\right)$,

$$
L(h(\mathbf{u}, \mathbf{v}))+12=L(h(\mathbf{u}, \mathbf{v}))+\sum_{i=1}^{12} L\left(h\left(\mathbf{u}, \mathbf{u}_{i}\right)\right) \leq 12 .
$$

This implies that $L(h(\mathbf{u}, \mathbf{v})) \leq 0$, so $\|\mathbf{u}-\mathbf{v}\| \geq 2 h_{0}$.

\section{1 graph classification problems}

Definition 1. Let $S^{2}$ be the sphere of radius 2, centered at $\mathbf{0}$. Let $\mathcal{V}$ be the set of packings $V \subset \mathbb{R}^{3}$ such that

1. $\operatorname{card}(V)=12$,

2. $V \subset S^{2}$,

3. $\|\mathbf{u}-\mathbf{v}\| \in\{0,2\} \cup[2.52,4]$ for all $\mathbf{u}, \mathbf{v} \in V$.

For each $V \in \mathcal{V}$, let $E_{\text {ctc }}$ be the contact graph on vertex set $V$; that is, the set of $\{\mathbf{u}, \mathbf{v}\} \subset V$ such that $\|\mathbf{u}-\mathbf{v}\|=2$.

Fejes Tóth's conjecture follows from the Inequality $\left(\left[L_{12}\right)\right.$, together with a proof that the classification of graphs $\left(V, E_{c t c}\right)$ with $V \in \mathcal{V}$ up to isomorphism contains exactly two graphs: the FCC contact graph and the HCP contact graph.

We formulate Inequality $\left(L_{12}\right)$ as a graph classification problem as well. The inequality holds trivially for a finite packing of cardinality at most 12 . For a contradiction, we may assume that $V$ belongs to the set of finite packings of cardinality at least 13, contained in a closed annulus of radii $\left[2,2 h_{0}\right]$ and that violate the inequality:

$$
\sum_{\mathbf{v} \in V} L(\|v\| / 2)>12 .
$$

Let $E_{\text {std }}$ be the set of edges $\{\mathbf{u}, \mathbf{v}\} \subset V$ such that $2 \leq\|\mathbf{u}-\mathbf{v}\| \leq 2 h_{0}$. The graph classification problem equivalent to $\left(L_{12}\right)$ is that the set of graphs $\left(V, E_{s t d}\right)$, with $V$ from this set of counterexamples, is empty.

In summary, the proof of Fejes Tóth's conjecture consists of two graph classification problems: one for the contact graphs $\left(V, E_{c t c}\right)$ involving vertex sets of cardinality 12 and one for the graphs $\left(V, E_{s t d}\right)$ involving vertex sets of cardinality at least 13 for the Inequality $\left(L_{12}\right)$. The proofs of these two classification results differ in detail, but the high-level structure is the same in both cases. The graphs are first represented combinatorially as hypermaps. (A hypermap is a finite set $D$ together with three permutations $e, n, f$ of $D$ that satisfy en $f=I$, the trivial permutation.) A computer program classifies the hypermaps satisfying given combinatorial properties obtained from the constraints imposed on the graphs $(V, E)$. Linear programs eliminate the extraneous cases; namely, those hypermaps that exist combinatorially but that do not admit a geometric realization. Finally, the inequalities used in the linear programs are proved by computer. 


\section{2 hypermap classification by computer}

The computer program that classifies hypermaps has been the subject of a exhaustive computer code formal verification project by G. Bauer and T. Nipkow [NBS06]. The original scope of the project was the set of graphs from the 1998 proof of the Kepler conjecture [HF06], but in 2010, Nipkow extended this work to include the classification of hypermaps needed for for the $L_{12}$ inequality. [1

\section{3 linear programs}

As mentioned above, linear programs eliminate the extraneous cases. The technology related to the linear programming has been significantly improved in the years following the proof of the Kepler conjecture. The thesis of S. Obua implemented the formal verification of linear programming proof certificates and used this to eliminate about $92 \%$ of the graphs that appear in the original proof of the Kepler conjecture [Obu08]. More recent work by Solvyev has optimized the formal verification of linear programs to such a degree that the speed of the formal verification of a linear program rivals the speed of the unverified execution of a linear program [SH11]. Work in progress by Solovyev intends to make a full formal verification of all linear programs needed to prove Inequality $\left(L_{12}\right)$.

The linear programs are generated in GLPK from an AMPL model that is independent of the hypermap. An OCaml program generates a separate AMPL data file for each linear program. When a single linear program fails to eliminate a hypermap, branch and bound methods are used to iteratively subdivide the domain into smaller pieces until linear programs are obtained that eliminate the hypermap. The process that was used to obtain a system of linear programming inequalities that works uniformly on all hypermaps was fully automated [Hal10]. In brief synopsis, when a linear program fails to eliminate a hypermap, two models of corresponding metric graph are compared, one based purely on the linear programming estimates of lengths and angles, and a second nonlinear model based on nonlinear relations between lengths and angles. A comparison of models is used to determine inadequacies in the linearization. This data is fed to a Mathematica program based on various heuristics to construct a candidate nonlinear inequality. The inequality is then shipped to the nonlinear optimization package CFSQP for extensive nonrigorous testing. From there, a formal specification of the inequality is generated in the proof assistant HOL Light. The formal specification is exported to program that uses interval arithmetic to verify inequalities by computer; and finally, the AMPL model is automatically updated with the new inequality. This process works remarkably well in practice to develop a small set of inequalities 2 that uniformly eliminate all undesired hypermaps.

\footnotetext{
${ }^{1}$ There are about 25,000 graphs that arise in the $L_{12}$ classification and only 8 graphs that arise in the contact graph. Because of the vast difference in complexity of these two classification problems, our discussion will focus on the $L_{12}$ classification.

${ }^{2}$ About 500 inequalities occur.
} 


\subsection{The classification of contact graphs}

As mentioned in an earlier footnote, there are about 25 thousand graphs that arise in the $L_{12}$ classification and only 8 graphs that arise in the contact graph classification. For the 8 graphs, it was not necessary to follow the lengthy linear programming procedure described in the previous subsection. This final subsection sketches a much simpler procedure to eliminate the unwanted cases. (Two of the eight possibilities are the HCP and FCC, and the other six cases are unwanted.)

Five of the six are eliminated with linear programming inequalities. The linear programs are based on the following simple inequalities:

1. The angles around each node sum to $2 \pi$.

2. The angle of each triangle in the contact graph equals $\arccos (1 / 3) \approx 1.23096$.

3. The opposite angles of each rhombus are equal.

4. Each angle of every rhombus is between 1.6292 and 2.16672 .

The final case is the graph shown in Figure 4 It is eliminated with the following observations. The perimeter of a spherical hexagon with sides $\pi / 3$ is $2 \pi$. However, the hexagons in the graph are spherically convex, and $2 \pi$ is a strict upper bound on the perimeter of a spherically convex hexagon. Thus, this case does not admit a geometric realization as a contact graph. Fejes Tóth's conjecture on sphere packings with kissing number twelve ensues.

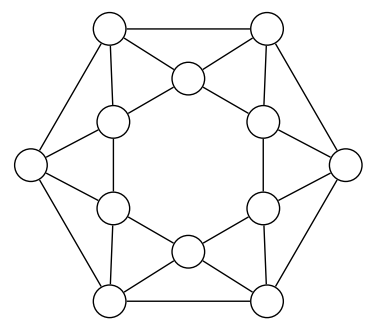

Fig. 4. This planar graph is not a contact graph.

\section{References}

Bez00. K. Bezdek, On a stronger form of Rogers' lemma and the minimum surface area of Voronoi cells in unit ball packings, J. Reine Angew. Math. 518 (2000), 131-143.

BV08. C. Bachoc and F. Vallentin, New upper bounds for kissing numbers from semidefinite programming, J. Amer. Math. Soc. 21 (2008), 909-924.

Fej43. L. Fejes Tóth, über die dichteste Kugellagerung, Mathematische Zeitschrift, vol. 48, 1943, pp. 676-684.

Fej69. _ Remarks on a theorem of R. M. Robinson, Studia Scientiarum Hungarica 4 (1969), 441-445. 
Fej72. __ Lagerungen in der Ebene auf der Kugel und im Raum, second ed., Springer-Verlag, Berlin-New York, 1972.

Fej89. , Research problems, Periodica Mathematica Hungarica 29 (1989), 89-91.

Hal10. T. C. Hales, Linear programs for the Kepler Conjecture, Mathematical Software ICMS 2010 (K. Fukuda, J. van der Hoeven, M. Joswig, and N. Takayama, eds.), Springer, September 2010.

Hal12a. , Dense sphere packings: a blueprint for formal proofs, London Math Soc. Lecture Note Series, vol. 400, Cambridge University Press, 2012.

Hal12b. _ A proof of Fejes Tóth's conjecture on sphere packings with kissing number twelve, arXiv:1209.6043, 2012.

HF06. T. C. Hales and S. P. Ferguson, The Kepler conjecture, Discrete and Computational Geometry 36 (2006), no. 1, 1-269.

HM10. T. C. Hales and S. McLaughlin, A proof of the dodecahedral conjecture, Journal of the AMS 23 (2010), 299-344, http://arxiv.org/abs/math/9811079

Mar09. C. Marchal, Study of the Kepler's conjecture: the problem of the closest packing, Mathematische Zeitschrift (2009).

NBS06. T. Nipkow, G. Bauer, and P. Schultz, Flyspeck I: Tame Graphs, International Joint Conference on Automated Reasoning (Ulrich Furbach and Natarajan Shankar, eds.), Lect. Notes in Comp. Sci., vol. 4130, Springer-Verlag, 2006, pp. 21-35.

Obu08. Stephen Obua, Flyspeck II: The basic linear programs, Ph.D. thesis, Technische Universität München, 2008.

Rog58. C. A. Rogers, The packing of equal spheres, Journal of the London Mathematical Society 3/8 (1958), 609-620.

SH11. A. Solovyev and T. C. Hales, Efficient formal verification of bounds of linear programs, LNCS, vol. 6824, pp. 123-132, Springer-Verlag, 2011. 